\title{
Novel quinazoline derivatives exhibit antitumor activity by inhibiting JAK2/STAT3
}

\author{
HAISHI QIAO ${ }^{1,2}$, DAN ZHAO $^{3}$, HENGFEI SHI ${ }^{1,2}$, KE REN $^{2},{\text { JIANXIN } \text { LI }^{3} \text { and ERGUANG LI }}^{1,2}$ \\ ${ }^{1}$ Medical School and State Key Laboratory of Pharmaceutical Biotechnology, Nanjing University; \\ ${ }^{2}$ Jiangsu Laboratory of Molecular Medicine, Medical School of Nanjing University; \\ ${ }^{3}$ School of Chemistry and Chemical Engineering and State Key Laboratory of Analytical Chemistry \\ for Life Science, Nanjing University, Nanjing, Jiangsu 210093, P.R. China
}

Received May 14, 2015; Accepted June 29, 2015

DOI: $10.3892 /$ or.2015.4140

\begin{abstract}
Quinazoline core-containing compounds such as gefitinib and erlotinib constitute an important group of antitumor drugs that act as receptor tyrosine kinase inhibitors against epidermal growth factor receptor (EGFR) kinase activity. We investigated a group of recently prepared 2-alkylsubstituted quinazolines (2-ASQs) for their antitumor activity against non-small cell lung carcinoma (NSCLC) cells. The compounds showed antitumor activity against A549, H1299, and $\mathrm{H} 460$ cells by induction of apoptosis. The $\mathrm{IC}_{50}$ values for (E)-2-propyl-4-styrylquinazoline (compound \#4) and (E)-2-cyclopropyl-4-styrylquinazoline (compound \#7) against these cell lines were 2-5 times lower than that of gefitinib. Unlike gefitinib that blocks EGFR phosphorylation, these compounds showed no activity against EGFR activation. Instead, the compounds suppressed both constitutive and IL-6-induced activation of JAK2/STAT3 phosphorylation and downstream gene expression. Transient expression of a constitutively active mutant of STAT3 reversed the pro-apoptotic effect of compound \#7. Using a nude mouse model bearing A549 xenografts, we showed that administration of \#7 at 15 and $30 \mathrm{mg} / \mathrm{kg}$ suppressed tumor growth. The present study therefore demonstrated that 2-alkyl substituted quinazolines target the JAK2/STAT3 pathway for their antitumor activity.
\end{abstract}

\section{Introduction}

Quinazoline derivatives have drawn significant attention ever since the discovery of gefitinib, a front line drug for lung

Correspondence to: Dr Erguang Li, Medical School, Nanjing University, Nanjing, Jiangsu 210093, P.R. China

E-mail: erguang@nju.edu.cn

Dr Jianxin Li, School of Chemistry and Chemical Engineering, Nanjing University, Nanjing, Jiangsu 210093, P.R. China

E-mail: lijxnju@nju.edu.cn

Key words: quinazolines, JAK2, STAT3, epidermal growth factor receptor, gefitinib, antitumor activity cancer. Several such as vandetanib, erlotinib and icotinib are important antitumor agents containing a quinazoline core. Gefitinib, also known as Iressa, is a synthetic anilinoquinazoline compound that acts as a receptor tyrosine kinase inhibitor (TKI) for its antitumor effect (1). Gefitinib selectively binds to the epidermal growth factor receptor (EGFR) tyrosine kinase domain through hydrogen bond formation of the $\mathrm{N}^{1}$ atom of the quinazoline core with the Met769 residue of the kinase domain, preventing ATP from binding and blocking subsequent signal transduction for their antitumor effect (1-3).

EGFR is a receptor tyrosine kinase that is overexpressed and occasionally mutated in cancers of epithelial origin, including non-small cell lung cancer (NSCLC), breast, colorectal and pancreatic cancer (4-6). Over $80 \%$ of lung squamous cell carcinomas and approximately half of all lung adenocarcinomas overexpress $\operatorname{EGFR}(7,8)$. EGFR expression is also associated with a poor response to therapy, development of cytotoxic drug resistance, disease progression and poor survival since the receptor tends to be hyperactivated $(9,10)$. Therefore, specific/selective inhibition of EGFR tyrosine kinase activity has demonstrated efficacy against certain types of tumors $(11,12)$.

One of the important signaling mediators downstream of EGFR signaling is signal transducer and activator of transcription-3 (STAT3). STAT3 belongs to a protein family of transcription factors which are essential for cellular functions $(13,14)$. In addition to EGFR, STAT3 can be activated by other receptors and non-receptor tyrosine kinases, such as the IL-6/gp130 receptor family, JAKs and Src kinases. STAT3 is persistently activated in a wide variety of tumors and has been detected in over $50 \%$ of NSCLC primary tumors and cell lines (15). STAT3 promotes tumor cell cycle progression, cell proliferation and tumor metastasis through differential gene regulation. In addition, aberrant STAT3 activation has been linked to gefitinib resistance $(15,16)$. Therefore, inhibitors of the JAK/STAT3 pathway have been developed as promising antitumor agents.

We have developed methods to prepare novel quinazolines (17,18), including ultrasound-promoted synthesis (19), and KOtBu-mediated stereoselective addition of quinazolines to alkynes (20). These methods have allowed the preparation 
of quinazolines with 2-position substitutions, including aryl groups and straight-, branched-chain, and cyclo-alkyl groups with moderate to good yields (20). These compounds have not been fully investigated for their biological activities. In view of the unique structural feature of 2-substitution and the rapid progress in preparation of 2-substituted quinazolines (21), we studied the antitumor activity of 2-alky substituted quinazolines (2-ASQs) and found that these novel compounds possessed anticancer activity against lung carcinoma cells. 2-ASQs showed no activity against EGFR activation, possibly due to the steric effect caused by a bulky group near the $\mathrm{N}^{1}$ atom. Instead, the compounds promoted apoptotic cell death through inhibition of STAT3 phosphorylation. In the present study, we report the antitumor activity of these newly synthesized quinazolines.

\section{Materials and methods}

Chemicals and reagents. Primary antibodies were commercially purchased from Cell Signaling Technology or from Santa Cruz Biotechnology. HRP-conjugated secondary antibodies were purchased from Sigma-Aldrich, and Alexa Fluor-conjugated secondary antibodies were purchased from Life Technologies. Gefitinib, AG490 and S3I-201 were purchased from Selleck and 3-(4,5-dimethylthiazol-2-yl)2,5-diphenyltetrazolium bromide (MTT) was obtained from Sigma-Aldrich. The preparation and identification of the quinozalines tested in the present study were described as previously reported (20).

Cell culture. A549, H460, H1299, 293T and Vero cells were purchased from the Cell Bank of the Chinese Academy of Sciences (Shanghai, China). A549, H460, 293T and Vero cells were cultured in high glucose Dulbecco's modified Eagle's medium (DMEM) supplemented with non-essential amino acids, $10 \%$ heat-inactivated fetal bovine serum (FBS), $2 \mathrm{mM}$ L-glutamine and sodium pyruvate (Life Technologies, Carlsbad, CA, USA). H1299 cells were cultured in RPMI-1640 supplemented with non-essential amino acids, $10 \%$ heatinactivated FBS, sodium pyruvate and $2 \mathrm{mM}$ L-glutamine. The cells were maintained at $37^{\circ} \mathrm{C}$ in a humidified atmosphere with $5 \% \mathrm{CO}_{2}$.

Cell viability assay. For cell culture studies, the compounds were dissolved in dimethylsulfoxide (DMSO) as a 1,000x stock and were diluted with culture medium before testing. The cytotoxic effect against Vero cells and $\mathrm{IC}_{50}$ values for tumor cells were measured by cell viability using the MTT assay. Briefly, the cells were seeded in 96-well plates at $5 \times 10^{3}$ cells/well in complete medium. The cells were treated without or with (DMSO at $0.1 \%$ as a control) a compound at different concentrations for $72 \mathrm{~h}$. At the end of the experiment, MTT was added to each well to a final concentration of $0.5 \mathrm{mg} / \mathrm{ml}$ for the measurement of formazan formation. The absorbance was measured at $570 \mathrm{~nm}$ in a VersaMax Microtiter Plate Reader (Molecular Devices, Sunnyvale, CA, USA). To obtain an $\mathrm{IC}_{50}$ value, cells in 96-well plates were treated with a test compound at concentrations of $0.3-30 \mu \mathrm{M}$ for $72 \mathrm{~h}$. Cell viability was then determined using MTT assay and the data were used to extrapolate $\mathrm{IC}_{50}$ values.
Western blot analysis. Cells were lysed in extraction buffer (300 mM NaCl, 1\% Nonidet P-40 in 20 mM Tris-HCl, pH 7.9) supplemented with protease and phosphatase inhibitors. The cell lysates were cleaned by centrifugation at 10,000 x g and soluble proteins were separated by SDS-PAGE, transferred to PVDF membranes and immunoblotted with primary antibodies followed by HRP-conjugated secondary antibodies and developed using an ECL Plus kit (GE Healthcare, Pittsburgh, PA, USA). The images were collected using Alpha Innotech FluorChem FC2 imaging system (Alpha Innotech, San Leandro, CA, USA).

Flow cytometric assay. The percentage of apoptotic cells was detected by FACS assay using an Annexin V-FITC/PI double labeling kit (Life Technologies). Briefly, the cells at a density of $3 \times 10^{5}$ cells $/ \mathrm{ml}$ were plated at $1.5 \mathrm{ml} /$ well in 6 -well plates and allowed to attach overnight. The cells were treated without or with a test compound at 1,3 and $10 \mu \mathrm{M}$ for $24 \mathrm{~h}$. The cells then were harvested and analyzed on FACSCalibur (BD Biosciences) following the manufacturer's instructions.

Immunofluorescence analysis. To determine pSTAT3 distribution, $\mathrm{H} 1299$ cells were pre-treated without or with $10 \mu \mathrm{M}$ compound \#7 and then stimulated with IL-6 (20 ng/ml) for $15 \mathrm{~min}$ at $37^{\circ} \mathrm{C}$. The cells were then fixed with $4 \%$ paraformaldehyde, followed by permeabilization with $0.2 \%$ Triton $\mathrm{X}-100$ in phosphate-buffered saline (PBS). The cells were incubated with anti-p-STAT3, followed by Alexa Fluor 568-conjugated secondary antibody (Life Technologies). Nuclei were stained with $10 \mu \mathrm{g} / \mathrm{ml}$ of DAPI for $15 \mathrm{~min}$. The images were captured on an Olympus FluoView FV10i confocal microscope.

Transfections. siRNAs against human STAT3 were purchased from GenePharma (Shanghai, China). Sequences of the oligos were as follows: sense, 5'-CCACTTTGGTGTTTCATAA-3'; siRNA and STAT3C were transfected using Lipofectamine 2000 (Life Technologies) following the manufacturer's instructions. Overexpression of STAT3C and STAT3 siRNA were verified after transfected for $24 \mathrm{~h}$ by western blotting.

STAT3 transcriptional activity. 293T cells were plated into a 12-well plate with $4 \times 10^{5}$ cells/well. The cells were transiently transfected with the p-STAT3-TA-luc plasmid (Beyotime, Nantong, China) using pRL as an internal control. Twenty four hours after transfection, the cells were then treated with or without \#7 for $2 \mathrm{~h}$ before treatment with IL- 6 for another $12 \mathrm{~h}$. The cells were lysed and used for luciferase analysis using a Dual-Luciferase Reporter Assay System and GloMax Luminometer (Promega Madison, WI, USA). The ratio of firefly luciferase activity over that of Renilla luciferase was used as a measurement of reporter gene activity.

Tumor xenograft study. Six-week-old female BALB/c nude mice were obtained from the Model Animal Research Center of Nanjing University (Nanjing, China). Animal care and experimental procedures were conducted in accordance with the guidelines of the Institutional Animal Care and Use Committee of the Medical School of Nanjing University. To initiate the xenografts, $2 \times 10^{6}$ of A549 cells in $0.15 \mathrm{ml}$ PBS were mixed with Matrigel at a 4:1 ratio (BD Biosciences) and 
A<smiles>[R1]C=Cc1nc([R2])nc2ccccc12</smiles>

2-substituted

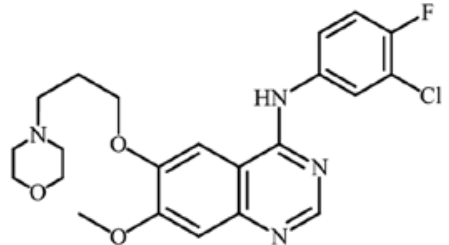

Gefitinib

\begin{tabular}{|c|c|c|c|c|}
\hline \# & $\mathrm{R}_{1}$ & $\mathrm{R}_{2}$ & $\operatorname{code}^{a}$ & activity $^{b}$ \\
\hline 1 & $\mathrm{Ph}$ & $\mathrm{Ph}$ & $3 a a$ & + \\
\hline 2 & 2-Thienyl & $\mathrm{Ph}$ & $3 \mathrm{ah}$ & ++ \\
\hline 3 & $\mathrm{Ph}$ & $4-\mathrm{CF}_{3}-\mathrm{Ph}$ & $3 \mathrm{ka}$ & - \\
\hline 4 & $\mathrm{Ph}$ & $n$-propyl & $3 \mathrm{pa}$ & +++++ \\
\hline 5 & $\mathrm{Ph}$ & $n$-henptyl & $3 q a$ & ++ \\
\hline 6 & $\mathrm{Ph}$ & $t$-butyl & $3 \mathrm{ra}$ & ++++ \\
\hline 7 & $\mathrm{Ph}$ & cyclopropyl & $3 \mathrm{sa}$ & +++++ \\
\hline 8 & $\mathrm{Ph}$ & cyclohexyl & 3 ta & + \\
\hline \multicolumn{4}{|c|}{ Gefitinib (Gef) } & ++++ \\
\hline
\end{tabular}

\section{C}

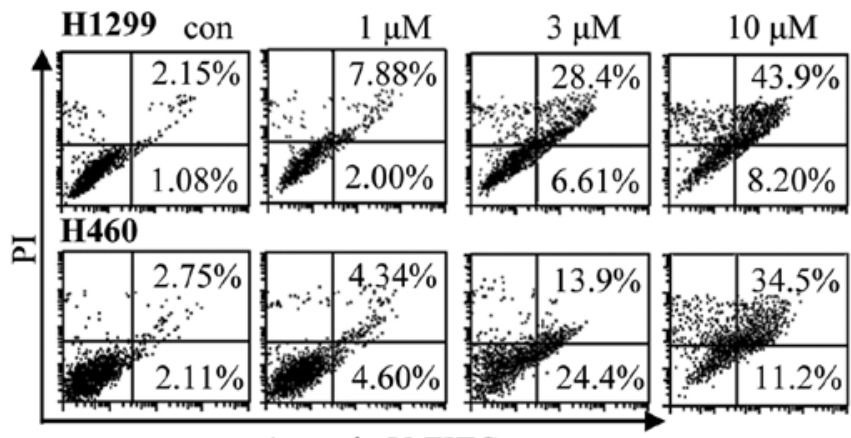

Annexin V-FITC
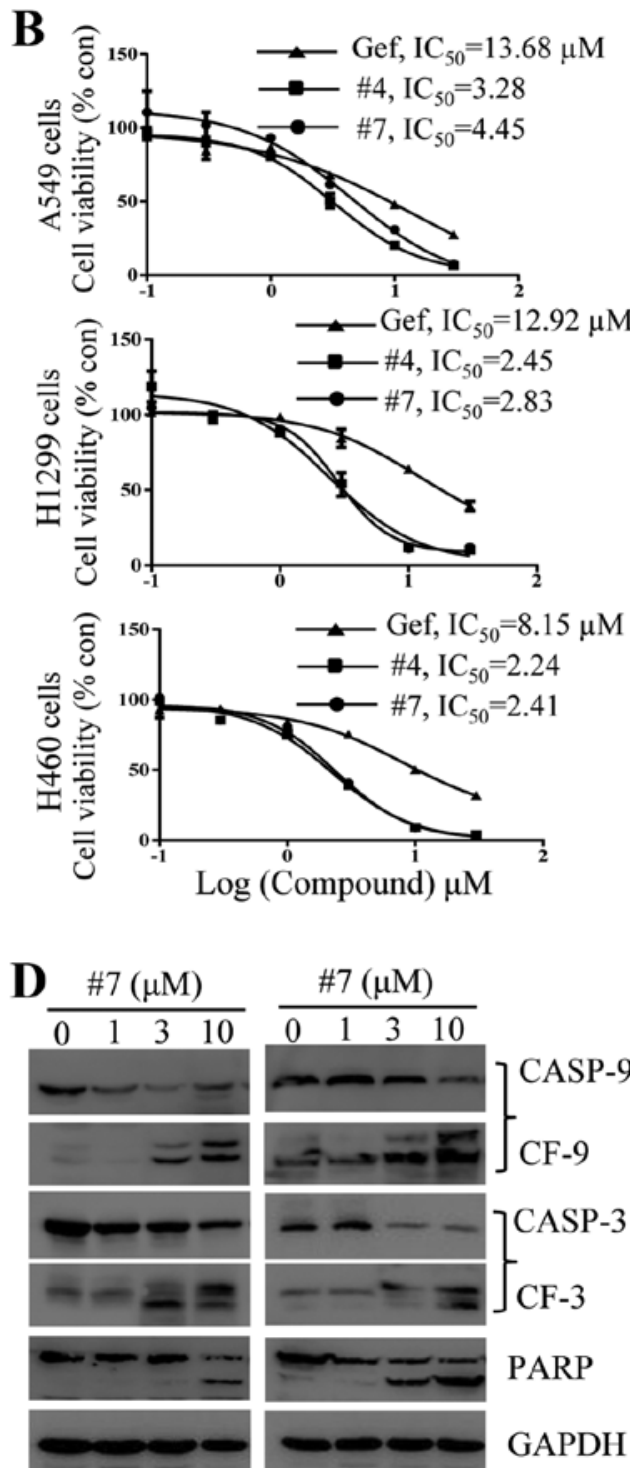

H1299 cells

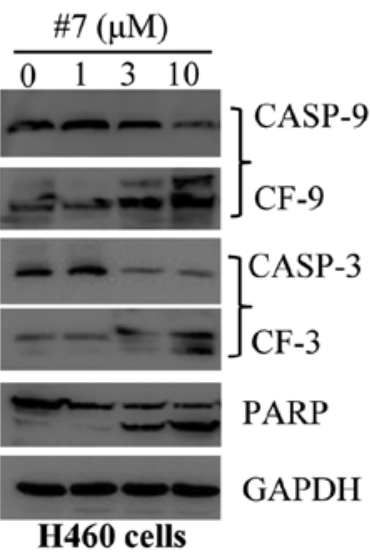

Figure 1. 2-Substituted quinazolines induce apoptotic cell death. (A) The chemical structures of the 2-substituted quinazolines and gefitinib and summary of results from an initial screening. For the initial screening, A549 lung carcinoma cells were treated with a single dose of a test compound at $30 \mu \mathrm{M}$, a concentration that showed no cytotoxic effect against non-cancerous Vero cells. Cell viability was determined using an MTT assay after $72 \mathrm{~h}$. ${ }^{\mathrm{a}}$ code, names used in Zhao et al (17); 'bactivity, inhibition of cell viability: -, no activity;,$+ \leq 20 \% ;++, \leq 40 \% ;+++, \leq 60 \% ;++++, \leq 80 \%$; and,$+++++>80 \%$. (B) Determination of the $\mathrm{IC}_{50}$ values of compound \#4 and \#7 for A549, H1299 or H460 cells. Gef, gefitinib. (C and D) Compound \#7 promotes cell death through apoptosis. H1299 or H460 cells were treated with $\# 7$ for $24 \mathrm{~h}$ at concentrations as indicated. (C) Cell apoptosis was determined by Annexin V/PI staining. (D) Caspase-3 and -9, and PARP cleavage were detected by immunoblot analysis.

were subcutaneously injected into the flank area of the nude mice. When palpable tumors were developed after 2 weeks, the mice were randomly divided into 4 groups, 6 in each group and treated by gavage with vehicle, with compound \#7 at 15 and $30 \mathrm{mg} / \mathrm{kg}$, or with genfitinb at $30 \mathrm{mg} / \mathrm{kg}$. The animals were treated 3 times/week for 4 weeks. The body weight of the mice and the size of the tumor mass were measured weekly. The tumor volume was calculated as (half of the length times the square of the width, in $\mathrm{mm}^{3}$ ). At the end of the experiment, the mice were sacrificed, and the tumors were excised and weighed. A portion of each tumor was fixed in buffered formalin and then embedded in paraffin for immunohistochemical (IHC) staining or for hematoxylin and eosin (H\&E) staining. The remaining tissue was stored at $-70^{\circ} \mathrm{C}$ for further analysis.
For Ki-67 and cleaved caspase-3 staining, tissue sections were de-paraffinized, fixed and stained with appropriate antibodies. At least three randomly selected fields were examined and photographed. The unfixed tumor tissues were homogenized and the proteins extracted were subjected to western blot analysis.

\section{Results}

The 2-substituted quinazolines exert antitumor effects by induction of cell apoptosis. The antitumor activity of the newly synthesized 2-ASQs and 2-aryl substituted quinazolines was first evaluated against 3 different cell lines with wild-type (wt) EGFR. The structures of the compounds tested are listed in Fig. 1A. Several 2-ASQs showed selective cytotoxic effects 

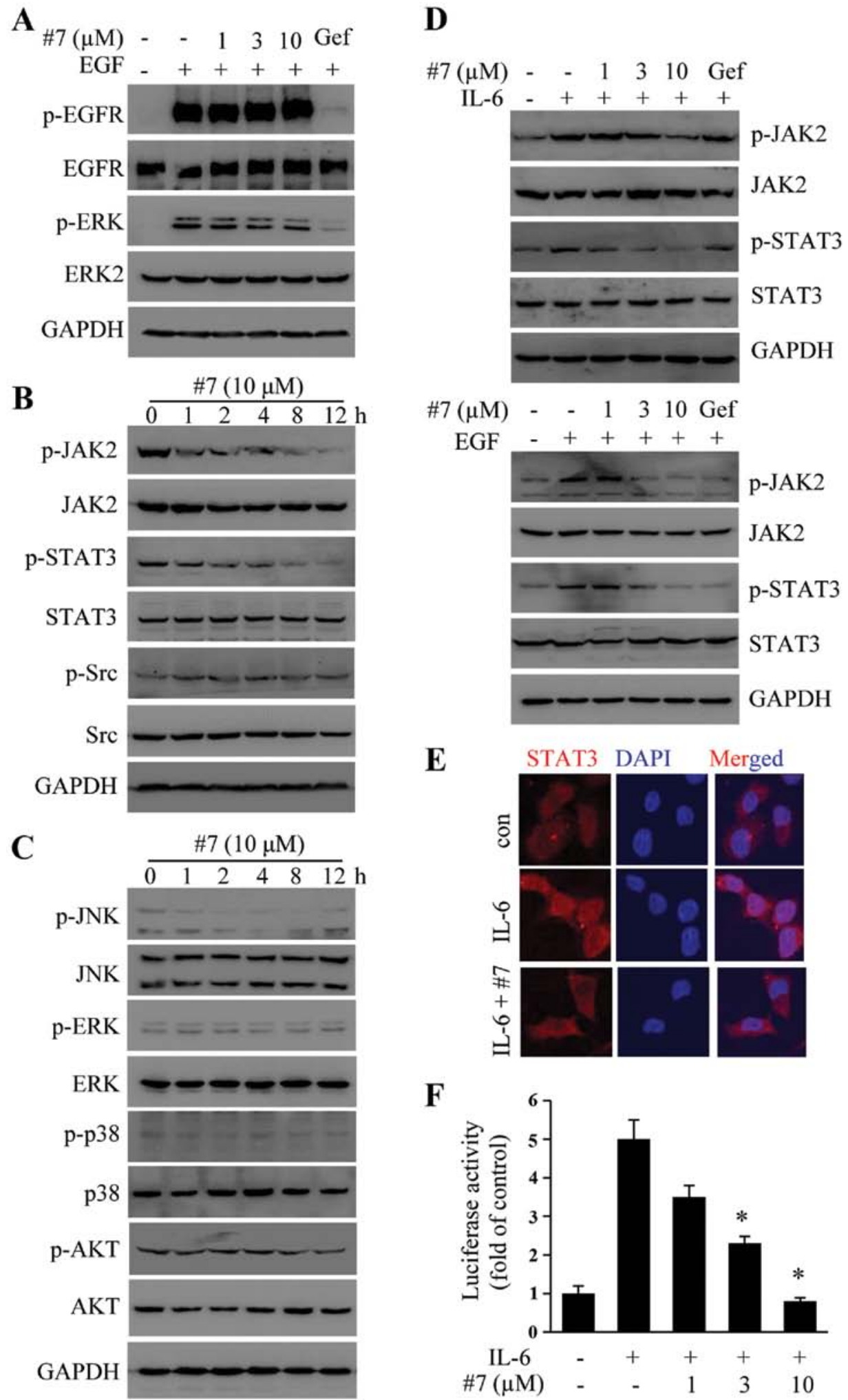

Figure 2. Effect of compound \#7 on EGFR and STAT3 phosphorylation and activation. (A) Compound \#7 shows no effect on EGFR activation. H1299 cells were pretreated with 1,3 and $10 \mu \mathrm{M}$ of $\# 7$ and $10 \mu \mathrm{M}$ gefitinib (Gef) for $2 \mathrm{~h}$ followed by treatment with $25 \mathrm{ng} / \mathrm{ml}$ EGF for 15 min. The phosphorylation status of EGFR (pY1068) and MAPK/ERK were analyzed by western blotting. (B) Compound \#7 blocks JAK2-STAT3 signaling. H1299 cells were treated with $10 \mu \mathrm{M}$ \#7 for the indicated times. Whole cell lysates were subjected to western blot analysis to detect both total and phosphorylated JAK2 (Y1007), Src (Tyr416) and STAT3. (C) Compound \#7 does not inhibit the AKT and MAPK pathway. H1299 cells were treated with $10 \mu \mathrm{M} \# 7$ for the indicated times. Whole cell lysates were subjected to western blot analysis to detect both total and phosphorylated AKT, JNK, p38 and ERK. (D) Effect of \#7 on ligand-induced STAT3 activation. $\mathrm{H} 1299$ cells were serum starved for $6 \mathrm{~h}$ and then pretreated with 1,3 and $10 \mu \mathrm{M} \# 7$ and $10 \mu \mathrm{M}$ Gef for $2 \mathrm{~h}$ followed by treatment with $20 \mathrm{ng} / \mathrm{ml}$ IL-6 (upper panel) or $25 \mathrm{ng} / \mathrm{ml} \mathrm{EGF} \mathrm{(lower} \mathrm{panel)} \mathrm{for} 15 \mathrm{~min}$. (E) Effect of \#7 on IL-6-induced STAT3 nuclear translocation. H1299 cells were serum starved for $6 \mathrm{~h}$ and then pretreated without or with $10 \mu \mathrm{M} \# 7$ for $2 \mathrm{~h}$ followed by treatment with $20 \mathrm{ng} / \mathrm{ml} \mathrm{IL-6}$ for $15 \mathrm{~min}$, and immunostained for STAT3 (red) and DAPI (blue). (F) Compound \#7 inhibits STAT3 transcription activity. 293T cells in triplicate were transiently transfected with p-STAT3-TA-luc using pRL as an internal control. After transfection for $24 \mathrm{~h}$, the cells were treated without or with $\# 7$ for $2 \mathrm{~h}$, followed by IL-6 (20 ng/ml) for $12 \mathrm{~h}$. The cells were lysed and used for luciferase analysis by measuring firefly and Renilla luciferase activities. The relative activity in the unstimulated samples was used as 1 . Bars represent means \pm SD of 3 independent experiments. Compound \#7 significantly blocked IL-6-induced firefly luciferase activity ( $\left.{ }^{*} \mathrm{P}<0.05\right)$. 
on tumor cells, while none of these compounds showed toxic effects on Vero cells after treatment for $72 \mathrm{~h}$ (Fig. 1A). We then performed experiments to quantitatively measure the cytotoxic effects of compound 4 (\#4) and 7 (\#7) on tumor cell growth by determining the half maximal inhibitory concentration $\left(\mathrm{IC}_{50}\right)$ values. A549, H460 and H1299 cells were cultured in the presence of varying concentrations of a test compound or gefitinib as a positive control. As shown in Fig. $1 \mathrm{~B}$, the $\mathrm{IC}_{50}$ values for \#4 and \#7 against these tumor cells were $~ 3$ - to 5-fold lower than that of gefitinib. We used \#7 as an example to delineate the mechanism of its antitumor activity. The more sensitive H460 and H1299 cells were incubated with \#7 at increased concentrations for $24 \mathrm{~h}$. Annexin V/PI double staining was used to assess apoptosis. As shown in Fig. 1C, treatment with \#7 significantly elevated the population of apoptotic cells The apoptosis was also validated with caspase activation determined by immunoblotting studies. As shown in Fig. 1D, in H460 and H1299 cells, treatment with \#7 dose-dependently caused caspase-9 and -3 , and PARP cleavage, indicating that \#7 exerted an antitumor effect through induction of cell apoptosis.

Compound \#7 does not inhibit EGF-induced EGFR phosphorylation. We sought to determine whether the antitumor activity of \#7 was associated with EGFR inhibition. As shown in Fig. 2A, gefitinib at $10 \mu \mathrm{M}$ completely blocked EGF-induced EGFR phosphorylation and downstream Erk activation. In contrast, pretreatment of H1299 cells with varying concentrations of \#7 showed no effect on EGFR activation (Fig. 2A) nor ERK, suggesting that the newly prepared 2-ASQs utilized a distinct mechanism to inhibit tumor cell growth compared with that of gefitinib.

Compound \#7 suppresses JAK2 and STAT3 phosphorylation and downstream signaling. STAT3 overexpression has been linked to tumorigenesis of NSCLC $(22,23)$. We then examined whether \#7 had the ability to inhibit STAT3 phosphorylation since both H460 and H1299 cells have hyperphosphorylated STAT3. As shown in Fig. 2B, H1299 has hyperphosphorylated STAT3 and JAK2. Treatment with $10 \mu \mathrm{M} \# 7$ for varying times inhibited both JAK2 (Y1007) and STAT3 (Tyr705) phosphorylation. For comparison, the treatment did not significantly affect Src (Y416) phosphorylation. In addition, \#7 showed no effect on Akt, p38, JNK or ERK phosphorylation (Fig. 2C).

We also sought to determine whether \#7 had an inhibitory effect on IL-6 and EGF-induced STAT3 phosphorylation. As shown in Fig. 2D, treatment with \#7 blocked both IL-6 and EGF-induced STAT3 and JAK2 phosphorylation. The effect of \#7 on STAT3 activation was confirmed by staining for IL-6-induced STAT3 nuclear translocation. As shown in Fig. 2E, treatment of H1299 cells with \#7 effectively blocked STAT3 nuclear translocation (Fig. 2E). In addition, we also demonstrated that \#7 blocked STAT3-mediated transcription activity as was determined using a reporter gene assay (Fig. 2F). These results together demonstrated that \#7 is a specific inhibitor of the STAT3 pathway.

Compound \#7 promotes apoptosis by inhibition of STAT3 signaling. We determined whether compound \#7-induced apoptosis was dependent on STAT3 inhibition. First, we examined the expression of anti-apoptosis protein expression
A

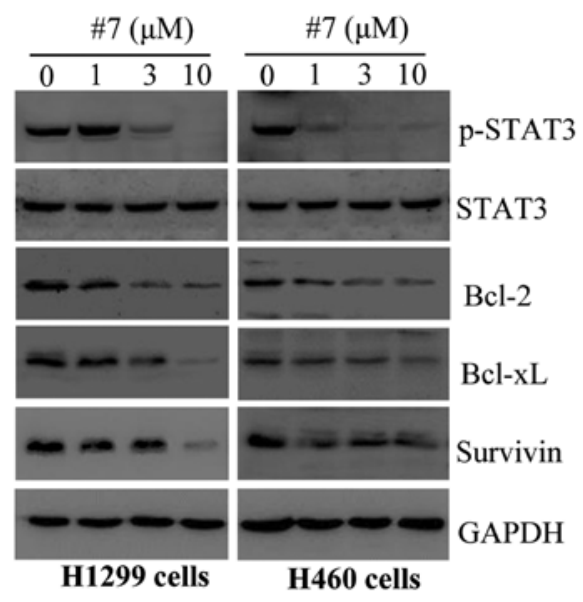

B

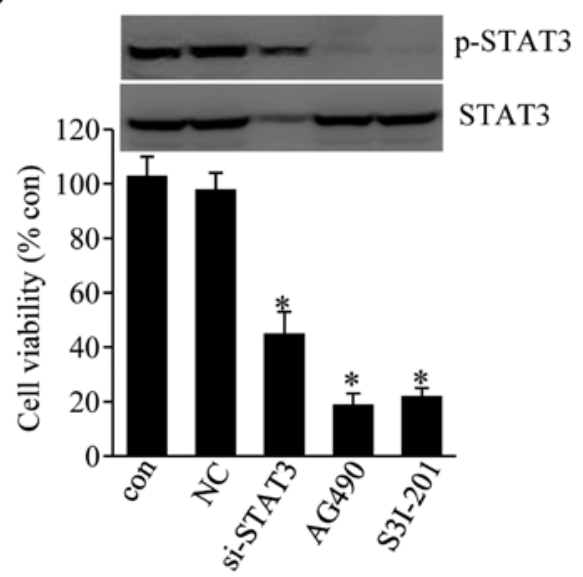

C

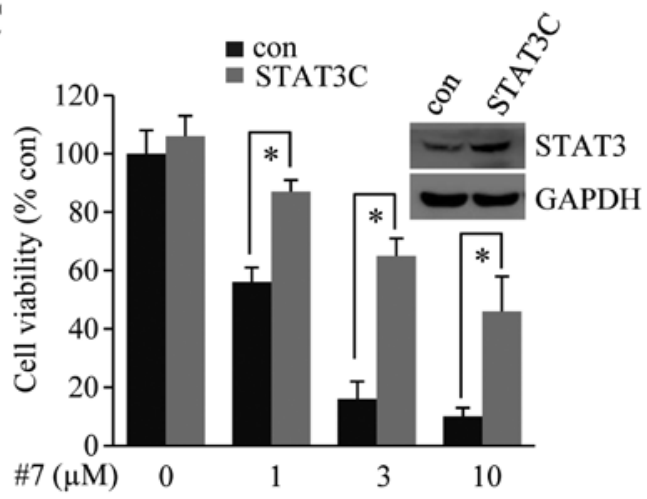

Figure 3. Compound \#7-induced apoptosis is dependent on STAT3 inhibition. (A) Compound \#7 prevents STAT3 phosphorylation and expression of related genes. H460 and H1299 cells were treated without or with \#7 at the indicated concentrations. The STAT3 phosphorylation and downstream gene expression were detected with a specific antibody by western blotting. (B) Effect of STAT3 siRNA or inhibitor on cell proliferation. H1299 cells were treated with STAT3 siRNA (si-STAT3), JAK2 inhibitor (AG490) or STAT3 inhibitor (S3I-201) for $72 \mathrm{~h}$. Cell viability was measured by MTT assay. The blots show STAT3 expression and phosphorylation in these cells, respectively. (C) Constitutively active mutant STAT3C reverses the effect of \#7-induced apoptosis. H1299 cells were transfected with empty vector (con) or STAT3C plasmid for $24 \mathrm{~h}$, and then treated with \#7 at the indicated concentrations for another $72 \mathrm{~h}$. Cell growth was measured by MTT assay. Bars represent means \pm SD of three independent experiments. Significant difference between STAT3C-expressing and vector controls $\left({ }^{*} \mathrm{P}<0.05\right)$.

in \#7-treated cells. As shown in Fig. 3A, treatment with \#7 resulted in reduced STAT3 phosphorylation in both the H1299 and $\mathrm{H} 460$ cells. The treatment also suppressed Bcl-2, Bcl-xL and survivin expression in these cells, indicating that \#7 
A

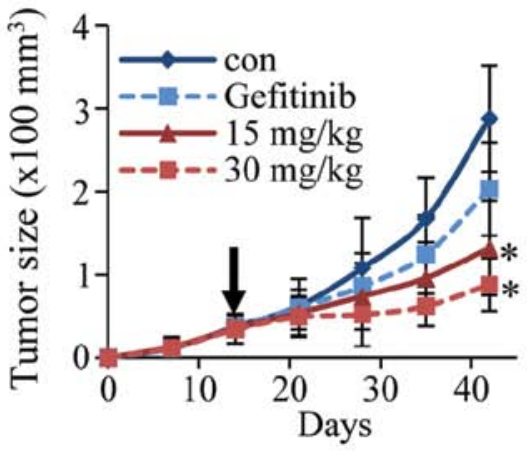

B

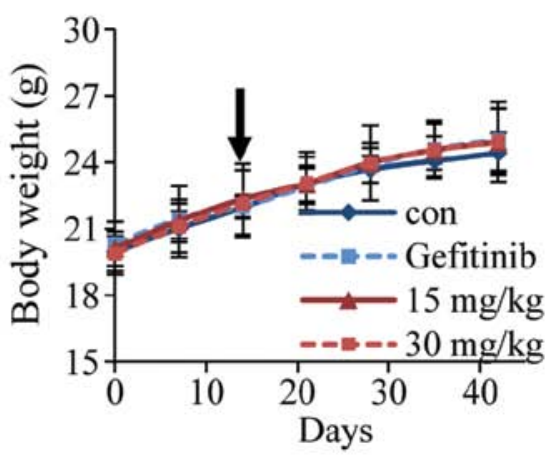

C

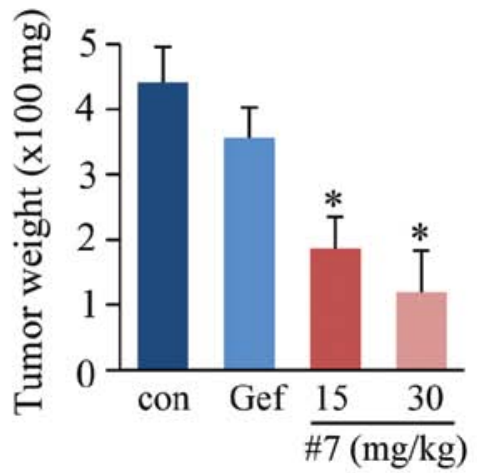

D

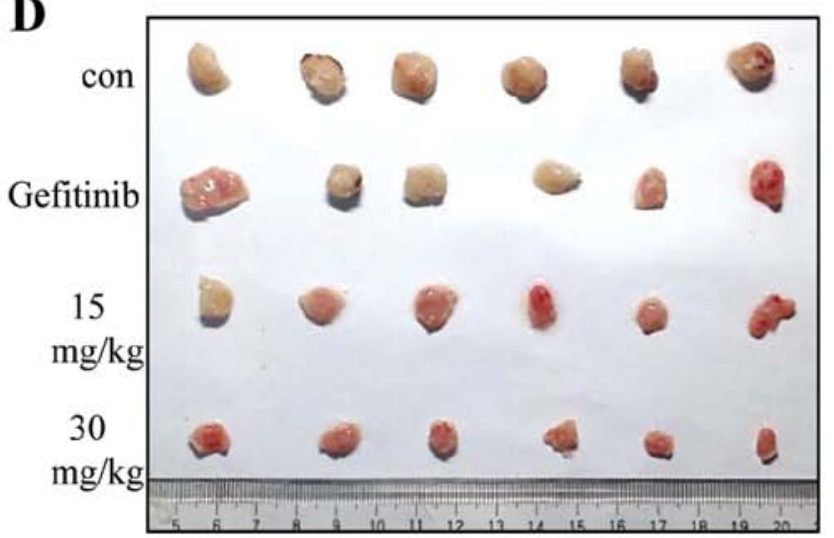

$\mathbf{E}$

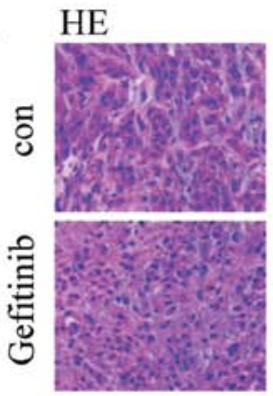

Ki-67 CF-3

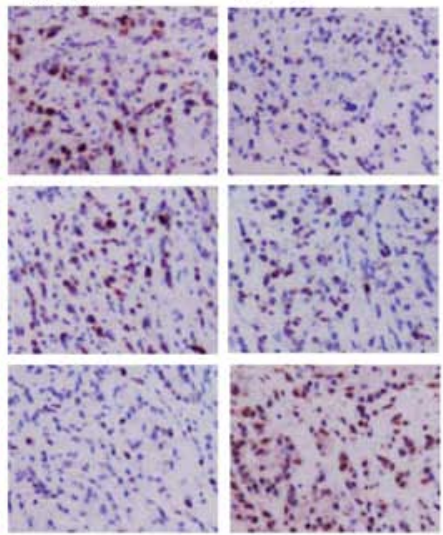

$\mathbf{F}$

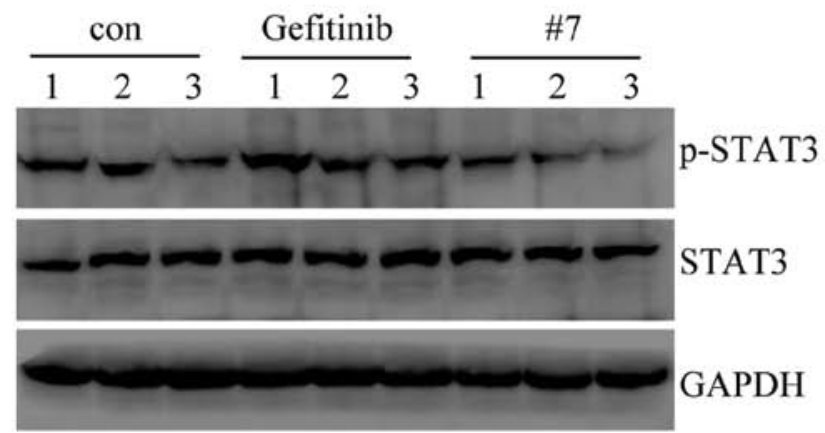

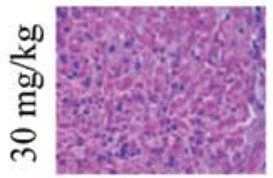

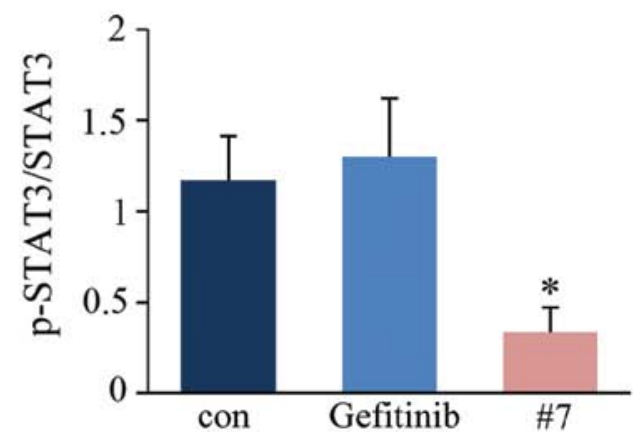

Figure 4. Inhibition of tumor growth in the animal model. (A) Average tumor volumes and (B) body weights measured weekly. A solid arrow indicates the beginning of the treatment. (C) Tumor weight and (D) images of the tumors excised at the end of the experiment. Each bar represents mean \pm SD ( $\mathrm{n}=6$ ). ${ }^{*} \mathrm{P}<0.05$; significant difference as compared with the control. (E) Immunohistochemical staining for cleaved caspase-3 and Ki-67 expression, and $\mathrm{H} \& \mathrm{E}$ staining of tumor samples. (F) Immunoblotting for p-STAT3 and STAT3 of excised tumor tissues using 3 animals $(1,2$ and 3 ) from each group (left panel). The intensities of the corresponding bands were quantitated using ImageJ software. The relative intensity of p-STAT3 to that of STAT3 in each group was averaged and plotted as average \pm standard deviation (right panel). ${ }^{*} \mathrm{P}<0.05$; significant difference as compared with the control.

promoted tumor cell apoptosis through inhibition of STAT3 signaling.

We then performed two separate experiments to substantiate the above conclusion. First, H1299 cells were transfected with an siRNA to knockdown STAT3 expression or were treated with specific inhibitors to block STAT3 phosphorylation. As shown in Fig. 3B, knockdown of STAT3 expression or inhibition of STAT3 phosphorylation resulted in significant reduction in cell viability. Conversely, we found that ectopic expression of a constitutively active mutant of STAT3 (STAT3C) was able to reverse the effect of \#7 on cell viability (Fig. 3C).
In vivo antitumor activity study in a xenograft model. The antitumor activity of \#7 was then evaluated in nude mice bearing xenografts. We chose to use the A549 xenograft since it is a well established animal for antitumor studies. BALB/c athymic nude mice bearing A549 tumors were treated with \#7 at 15 and $30 \mathrm{mg} / \mathrm{kg}$, with gefitinib at $30 \mathrm{mg} / \mathrm{kg}$, or with PBS by intragastric gavage. After treatment for 4 weeks, the mice were sacrificed and the size of the tumors was recorded. As shown in Fig. 4D, tumors in mice treated with \#7 at 15 and $30 \mathrm{mg} / \mathrm{kg}$ were significantly smaller than tumors in the control group. The dynamic change in tumor size also demonstrated that \#7 markedly attenuated A549 tumor growth in 
nude mice without significantly affecting the body weight of the animals (Fig. 4A-C). Examination after H\&E staining revealed that the tumor tissues from the $30 \mathrm{mg} / \mathrm{kg}$ group were filled with fibrous tissues accompanied by reduced numbers of cancer cells (Fig. 4E). The level of Ki-67, a marker of cell proliferation detected by immunohistochemical staining, was decreased in the treated groups compared to that in the untreated controls. In contrast, the level of cleaved caspase-3 was increased in the \#7-treated tumors, indicating that \#7 promoted tumor cell apoptosis (Fig. 4E). We also examined the STAT3 phosphorylation status in the tumor tissues by western blot analysis. Consistent with the results from the cell culture studies, treatment with \#7 also resulted in decreased phosphorylation of STAT3 in these samples (Fig. 4F).

Taken together, these results demonstrated that 2-ASQ derivatives exert antitumor effects through STAT3 inhibition.

\section{Discussion}

Quinazoline derivatives have demonstrated distinct therapeutic activities including anticancer, anti-inflammatory, antibacterial, antiviral and antiobesity effects (21). We previously prepared a broad range of quinazoline derivatives and characterized compounds \#4 and \#7 as having potent antitumor activity through inhibition of JAK2/STAT3 activation. Several small organic molecules including ursolic acid and curcumin have been identified as inhibitors of STAT3 pathway signaling $(24,25)$. Most of the JAK1/2 inhibitors that are approved for clinical use or testing are heterocyclic compounds including those containing a pyrimidine or pyrrolopyrimidine ring $(26,27)$. It is known that quinazolines block EGFR activity by blocking ATP binding to the kinase domain. Although we do not know the targets of 2-ASQ action, our data and data published by other groups strongly suggest that the JAK/STAT3 pathway is a potential target of their action. In a recent docking study, Yang et al (28) predicted that quinazoline derivatives also could interact with the active site of JAK2 and selectively block JAK2 activity. In addition, 2-guanidinosubstituted quinazolines have recently been identified as new inhibitors of the STAT3 pathway (29). Further studies to optimize the structures of the lead compounds and identify their molecular targets are critical considering the importance of the role of the JAK/STAT3 pathway in tumor development and antitumor resistance to TKIs.

Nearly all patients develop resistance to TKIs and relapse for a variety of reasons $(30,31)$. Given the fact that $\sim 50 \%$ of tumors possess activated STAT3 and only $16 \%$ contain activating EGFR mutations that have increased sensitivity to EGFR TKIs (10), it is possible that further study of 2-ASQs may lead to potent chemotherapeutics targeting STAT3 activation. We prepared 2-ASQs with relatively high yields under mild conditions. 2-ASQ derivatives with further modifications can also be readily prepared with methods developed by us. STAT3 signaling is frequently activated, often constitutively, in a variety of human malignancies, including cancers of the head and neck, colorectum, cervix, breast and esophagus. STAT3 regulates cancer progression including cell proliferation, apoptosis resistance and angiogenesis. As JAK/STAT signaling controls a variety of cellular processes and affects the microenvironment of tumor growth and antitumor immu- nity, therapeutics targeting the JAK/STAT3 pathway may have a wide range of systemic ramifications for tumor treatment and inflammatory diseases.

\section{Acknowledgements}

The authors would like to thank Dr Xuancheng Guo for proofreading the manuscript. The present study was supported financially by grants from NSFC of China (grant nos. 81121062 and 81371772).H.S.Q. was a recipient of a Predoctoral Fellowship from Jiangsu Innovation Programs for Graduate Students.

\section{References}

1. Herbst RS, Fukuoka M and Baselga J: Gefitinib - a novel targeted approach to treating cancer. Nat Rev Cancer 4: 956-965, 2004.

2. Wheeler DL, Dunn EF and Harari PM: Understanding resistance to EGFR inhibitors - impact on future treatment strategies. Nat Rev Clin Oncol 7: 493-507, 2010.

3. Yun CH, Boggon TJ, Li Y, Woo MS, Greulich H, Meyerson M and Eck MJ: Structures of lung cancer-derived EGFR mutants and inhibitor complexes: Mechanism of activation and insights into differential inhibitor sensitivity. Cancer Cell 11: 217-227, 2007.

4. Agus DB, Akita RW, Fox WD, Lewis GD, Higgins B, Pisacane PI, Lofgren JA, Tindell C, Evans DP, Maiese K, et al: Targeting ligand-activated ErbB2 signaling inhibits breast and prostate tumor growth. Cancer Cell 2: 127-137, 2002.

5. Baselga J, Rischin D, Ranson M, Calvert H, Raymond E, Kieback DG, Kaye SB, Gianni L, Harris A, Bjork T, et al: Phase I safety, pharmacokinetic, and pharmacodynamic trial of ZD1839, a selective oral epidermal growth factor receptor tyrosine kinase inhibitor, in patients with five selected solid tumor types. J Clin Oncol 20: 4292-4302, 2002.

6. Scagliotti GV, Selvaggi G, Novello S and Hirsch FR: The biology of epidermal growth factor receptor in lung cancer. Clin Cancer Res 10: 4227s-4232s, 2004.

7. Maurizi M, Almadori G, Ferrandina G, Distefano M, Romanini ME, Cadoni G, Benedetti-Panici P, Paludetti G, Scambia $G$ and Mancuso S: Prognostic significance of epidermal growth factor receptor in laryngeal squamous cell carcinoma. $\mathrm{Br}$ J Cancer 74: 1253-1257, 1996.

8. Tateishi M, Ishida T, Kohdono S, Hamatake M, Fukuyama Y and Sugimachi K: Prognostic influence of the co-expression of epidermal growth factor receptor and c-erbB-2 protein in human lung adenocarcinoma. Surg Oncol 3: 109-113, 1994.

9. Robertson SC, Tynan J and Donoghue DJ: RTK mutations and human syndromes: When good receptors turn bad. Trends Genet 16: 368, 2000.

10. Rosell R, Moran T, Queralt C, Porta R, Cardenal F, Camps C, Majem M, Lopez-Vivanco G, Isla D, Provencio M, et al; Spanish Lung Cancer Group: Screening for epidermal growth factor receptor mutations in lung cancer. N Engl J Med 361: 958-967, 2009.

11. Robinson KW and Sandler AB: EGFR tyrosine kinase inhibitors: Difference in efficacy and resistance. Curr Oncol Rep 15: 396-404, 2013.

12. Zhong D, Ru Y, Wang Q, Zhang J, Zhang J, Wei J, Wu J, Yao L, $\mathrm{Li} \mathrm{X}$ and $\mathrm{Li} \mathrm{X}$ : Chimeric ubiquitin ligases inhibit non-small cell lung cancer via negative modulation of EGFR signaling. Cancer Lett 359: 57-64, 2015.

13. Zhang L, Pan J, Dong Y, Tweardy DJ, Dong Y, Garibotto G and Mitch WE: Stat3 activation links a C/EBPS to myostatin pathway to stimulate loss of muscle mass. Cell Metab 18: 368-379, 2013.

14. Tierney MT, Aydogdu T, Sala D, Malecova B, Gatto S, Puri PL, Latella L and Sacco A: STAT3 signaling controls satellite cell expansion and skeletal muscle repair. Nat Med 20: 1182-1186, 2014.

15. Gao SP, Mark KG, Leslie K, Pao W, Motoi N, Gerald WL, Travis WD, Bornmann W, Veach D, Clarkson B, et al: Mutations in the EGFR kinase domain mediate STAT3 activation via IL-6 production in human lung adenocarcinomas. J Clin Invest 117: 3846-3856, 2007. 
16. Wu K, Chang Q, Lu Y, Qiu P, Chen B, Thakur C, Sun J, Li L, Kowluru A and Chen F: Gefitinib resistance resulted from STAT3-mediated Akt activation in lung cancer cells. Oncotarget 4: 2430-2438, 2013.

17. Zhao D, Zhu MX, Wang Y, Shen Q and Li JX: Pd(0)-catalyzed benzylic arylation-oxidation of 4-methylquinazolines via $\mathrm{sp}^{3}$ $\mathrm{C}-\mathrm{H}$ activation under air conditions. Org Biomol Chem 11: 6246-6249, 2013.

18. Zhao D, Wang T, Shen Q and Li JX: $n-\mathrm{Bu}_{4} \mathrm{NI}-$ catalyzed selective dual amination of $\mathrm{sp}^{3} \mathrm{C}-\mathrm{H}$ bonds: Oxidative domino synthesis of imidazo[1,5-c]quinazolines on a gram-scale. Chem Commun 50 4302-4304, 2014.

19. Zhang L, Gao Z, Peng C, Bin ZY, Zhao D, Wu J, Xu Q and Li JX: Ultrasound-promoted synthesis and immunosuppressive activity of novel quinazoline derivatives. Mol Divers 16: 579-590, 2012.

20. Zhao D, Shen Q, Zhou YR and Li JX: KO $t$ Bu-mediated stereoselective addition of quinazolines to alkynes under mild conditions. Org Biomol Chem 11: 5908-5912, 2013.

21. Wang D and Gao F: Quinazoline derivatives: Synthesis and bioactivities. Chem Cent J 7: 95, 2013.

22. Yu H and Jove R: The STATs of cancer - new molecular targets come of age. Nat Rev Cancer 4: 97-105, 2004.

23. Harada D, Takigawa N and Kiura K: The role of STAT3 in non-small cell lung cancer. Cancers 6: 708-722, 2014.

24. Pathak AK, Bhutani M, Nair AS, Ahn KS, Chakraborty A Kadara H, Guha S, Sethi G and Aggarwal BB: Ursolic acid inhibits STAT3 activation pathway leading to suppression of proliferation and chemosensitization of human multiple myeloma cells. Mol Cancer Res 5: 943-955, 2007.
25. Bharti AC, Donato N and Aggarwal BB: Curcumin (diferuloylmethane) inhibits constitutive and IL-6-inducible STAT3 phosphorylation in human multiple myeloma cells. J Immunol 171: 3863-3871, 2003.

26. Baskin R, Majumder A and Sayeski PP: The recent medicinal chemistry development of Jak2 tyrosine kinase small molecule inhibitors. Curr Med Chem 17: 4551-4558, 2010.

27. Siveen KS, Sikka S, Surana R, Dai X, Zhang J, Kumar AP, Tan BK, Sethi G and Bishayee A: Targeting the STAT3 signaling pathway in cancer: Role of synthetic and natural inhibitors. Biochim Biophys Acta 1845: 136-154, 2014.

28. Yang SH, Khadka DB, Cho SH, Ju HK, Lee KY, Han HJ, Lee KT and Cho WJ: Virtual screening and synthesis of quinazolines as novel JAK2 inhibitors. Bioorg Med Chem 19: 968-977, 2011.

29. LaPorte MG, da Paz Lima DJ, Zhang F, Sen M, Grandis JR, Camarco D, Hua Y, Johnston PA, Lazo JS, Resnick LO, et al: 2-Guanidinoquinazolines as new inhibitors of the STAT3 pathway. Bioorg Med Chem Lett 24: 5081-5085, 2014.

30. Pao W, Miller VA, Politi KA, Riely GJ, Somwar R, Zakowski MF, Kris MG and Varmus H: Acquired resistance of lung adenocarcinomas to gefitinib or erlotinib is associated with a second mutation in the EGFR kinase domain. PLoS Med 2: e73, 2005.

31. Engelman JA and Jänne PA: Mechanisms of acquired resistance to epidermal growth factor receptor tyrosine kinase inhibitors in non-small cell lung cancer. Clin Cancer Res 14: 2895-2899, 2008. 